Rapid Reviews COVID-19

\title{
Review 2: "Differential \\ effects of antiseptic mouth \\ rinses on SARS-CoV-2 \\ infectivity in vitro"
}

\section{Chaminda Seneviratne ${ }^{1}$}

${ }^{1}$ Associate Professor, Duke National University of Singapore, National Dental Centre Singapore

Published on: Jan 30, 2021

DOI: $10.1162 / 2 \mathrm{e} 3983 f 5 . e 898 f a 45$

License: Creative Commons Attribution 4.0 International License(CC-BY 4.0) 


\section{$\underline{\text { RR:C19 Evidence Scale rating by reviewer: }}$}

- Potentially informative. The main claims made are not strongly justified by the methods and data, but may yield some insight. The results and conclusions of the study may resemble those from the hypothetical ideal study, but there is substantial room for doubt. Decision-makers should consider this evidence only with a thorough understanding of its weaknesses, alongside other evidence and theory. Decisionmakers should not consider this actionable, unless the weaknesses are clearly understood and there is other theory and evidence to further support it.

*****************************************

\section{Review:}

This in-vitro study set out to investigate whether the efficacy of various commercially available mouth-rinses is due to cytotoxicity or true anti-viral activity. Considering the laboratory nature of the study, the scientific method to investigate this objective is appropriate. The manuscript is novel, and sheds light on an area not previously investigated. However, there are various limitations which should be highlighted before extrapolating the findings to clinical settings. According to the in vitro results, Colgate peroxyl and Povidone-Iodine have shown to possess a higher cytotoxicity than Listerine and chlorhexidine gluconate (CHG). However, Colgate peroxyl and PovidoneIodine-based mouth-rinses have been safely used in dental and oral applications for decades. If repurposing of these mouth-rinses in COVID-19 patients can help decrease the SARS Co-V2 viral load through oral route, cytotoxicity-induced antiviral effect can be overruled. Even though the authors have tried to minimize the mouth rinseassociated cytotoxicity by experimental handling, in a real-life scenario the cytotoxicity is expected to be much more reduced as the oral cavity is bathed continuously in saliva and food. Moreover, application of mouth-rinses in real-life is limited to a very short duration (e.g. 30 seconds) compared to the laboratory settings. Therefore, it is not possible to conclude that Listerine and CHG to be better mouth-rinse products for SARS Co-V2 infection. Further tissue-level experiments with animal models as well as clinical trials in patients are needed for firm conclusions. Mouth-rinses may have a potential application in reducing the salivary SARS CoV-2 level under certain conditions. In this regard, the current study provides valuable insight and data for future work on this area. 